\title{
Steady-State Diffusion of Water through Soft-Contact-Lens Materials
}

\author{
Fornasiero F., Krull F., ${ }^{1}$ Prausnitz J. M. and Radke C. J. ${ }^{*}$
}

\author{
Chemical Engineering Department \\ University of California, Berkeley, CA 94720-1462 \\ and \\ Chemical and Earth Sciences Divisions \\ Lawrence Berkeley National Laboratory \\ Berkeley, CA 94720.
}

Submitted to Biomaterials

November 18, 2004

Revised

January 31, 2005

1 Present address: Department of Chemical Engineering at RWTH, Turmstraße 46, 52056 Aachen, Germany, Email: krull@ivt.rwth-aachen.de

* Corresponding author: TEL (510) 642-5204; FAX 510-642-4778; email radke@,berkeley.edu 


\section{Abstract}

Water transport through soft contact lenses (SCL) is important for acceptable performance on the human eye. Chemical-potential gradient-driven diffusion rates of water through soft-contact-lens materials are measured with an evaporation-cell technique. Water is evaporated from the bottom surface of a lens membrane by impinging air at controlled flow rate and humidity. The resulting weight loss of a water reservoir covering the top surface of the contact-lens material is recorded as a function of time.

New results are reported for a conventional hydrogel material (SofLens ${ }^{\mathrm{TM}}$ One Day, hilafilcon A, water content at saturation $w_{10}=70$ weight $\%$ ) and a silicone hydrogel material (PureVision ${ }^{\mathrm{TM}}$, balafilcon $\mathrm{A}, w_{10}=36 \%$ ), with and without surface oxygen plasma treatment. Also, previously reported data for a conventional HEMA-SCL $\left(w_{10}=\right.$ $38 \%$ ) hydrogel are reexamined and compared with those for SofLens ${ }^{\mathrm{TM}}$ One Day and PureVision $^{\mathrm{TM}}$ hydrogels. Measured steady-state water fluxes are largest for SofLens ${ }^{\mathrm{TM}}$ One Day, followed by PureVision ${ }^{\mathrm{TM}}$ and HEMA. In some cases, the measured steadystate water fluxes increase with rising relative air humidity. This increase, due to an apparent mass-transfer resistance at the surface (trapping skinning), is associated with formation of a glassy skin at the air/membrane interface when the relative humidity is below $55-75 \%$.

Steady-state water-fluxes are interpreted through an extended Maxwell-Stefan diffusion model for a mixture of species starkly different in size. Thermodynamic nonideality is considered through Flory-Rehner polymer-solution theory. Shrinking/swelling is self-consistently modeled by conservation of the total polymer mass. Fitted MaxwellStefan diffusivities increase significantly with water concentration in the contact lens. 
Keywords: evaporation-cell method, water diffusion-coefficient, soft contact lens, extended Maxwell-Stefan model, trapping skinning, glass transition.

\section{Introduction}

The performance of soft contact lenses (SCL) on the human eye depends strongly on the water content and water-transport properties of the contact lens [1]. There is much evidence to believe that in-vivo contact-lens dehydration is primarily caused by an evaporative-dehydration process [2-7]. Evaporation at the anterior surface of a partially dewetted SCL draws water from the post-lens tear film (PoLTF) and through the lens into the environment (air). If this outward water transport rate is sufficiently large, the PoLTF may deplete, causing corneal desiccation $[8,9]$, reduced on-eye movement $[10,11]$ and, in extreme cases, lens adherence to the cornea surface [7, 12]. Water loss may also lead to discomfort and to dryness symptoms [13], and may reduce oxygen transmissibility [14], as well as fitting and corrective power of the lens [7]. Transport of water through a SCL can also be induced by an osmotic gradient between pre-lens and post-lens tear films [15], or by mechanical lid stress [16]. Understanding the importance of these phenomena requires quantitative knowledge of the rate of water transport through a thin polymer membrane.

Previous work on water diffusion in soft contact lenses has focused primarily on measuring self-diffusion coefficients [17-22], which characterize water transport in the absence of a chemical potential gradient. A few studies report measurements of kinetic sorption/desorption [23-27] of water or steady-state water fluxes $[8,28]$ driven by a water-activity gradient through a SCL. 
In this work, we report steady-state diffusion rates of water through soft-contactlens materials under a water chemical-potential gradient. To measure water flux precisely, we have greatly improved a recently developed evaporation-cell method [28]. In this experimental method, a water activity-gradient is established by water evaporation from the bottom surface of a SCL thin membrane whose upper surface is maintained in contact with pure water. In a recent report [29], we have shown that our method gives reliable diffusivities by comparing evaporation-cell data with results from other techniques for a HEMA SCL. Here, we study a conventional hydrogel (SofLens ${ }^{\mathrm{TM}}$ One Day) and a silicon hydrogel (PureVision ${ }^{\mathrm{TM}}$ ) with (prefix ST-) and without (prefix NT-) surface plasma treatment. For comparison, we also reconsider the previous results obtained for a HEMA SCL [29] .

Two distinct frameworks are commonly used to describe solute transport through membranes: the pore-flow model and the solution-diffusion model [30]. In the pore-flow framework, the membrane polymer is viewed as a porous solid matrix through which the solute hydraulically flows, whereas in the solution-diffusion model the polymer/solute is viewed as a molecular mixture, and solute transport is via diffusion. According to Wijmans and Baker [30], in a solution-diffusion membrane, the free-volume elements are a consequence of statistical concentration fluctuations in the membrane. They appear and disappear in about the same time scale as that for the motion of the solute penetrant. Conversely, in a porous-medium membrane, the free volume elements (pores) are relatively stable and do not fluctuate in position and size on the time scale of penetrant transport. In contrast to others who adopt a porous-medium model to describe water transport through SCL materials $[2,31]$, we argue that the solution-diffusion model 
provides a more consistent representation of the physics of water transport in SCL hydrogel materials. Further, the very small pressure-induced water permeabilities of HEMA $[32,33]$ and PureVision ${ }^{\mathrm{TM}}$ SCL [32] support a diffusive rather than a pressuredriven viscous mechanism for water transport through these materials.

Within the solution-diffusion framework, Fick's law is generally adopted to describe diffusion in binary systems because of its simplicity. However, Fick's law does not explicitly account for thermodynamic nonideality and also cannot be readily applied to multicomponent systems. We view a water/hydrogel SCL as a highly nonideal molecular mixture. Moreover, we are interested in extending our present study to include simultaneous, multicomponent transport of other small molecules (e.g., salt or oxygen) through a contact lens. A self-consistent description of multicomponent transport for a simple fluid mixture is given by the generalized Maxwell-Stefan (GMS) framework that accounts both for thermodynamic nonideality and for diffusion coupling in multicomponent mixtures [34]. However, even in the binary case, difficulties are encountered when GMS is applied to transport through a cross-linked polymeric membrane because the unknown molecular weight (or, equivalently, a molar concentration) of the membrane must be specified [28]. This problem arises because GMS does not represent correctly the frictional resistance between molecules that differ greatly in size. Recently, we have proposed an extended Maxwell-Stefan model (EMS) to describe multicomponent transport for molecules of starkly different size [35]. When EMS is applied to solute transport in a membrane, there is no need to specify the unknown membrane molecular weight. In this work, we adopt the EMS model to analyze our experimental water fluxes through SCL materials. Swelling/shrinkage of the 
membrane is included self-consistently by imposing conservation of the polymer mass in the membrane. For completeness, we report also Fickian diffusion coefficients for water in our SCL materials.

\section{Experimental}

\section{Materials}

Distilled/deionized water is obtained from a MilliQ ${ }^{\mathrm{TM}}$ purification system; water resistivity is greater than $18 \mathrm{M} \Omega \mathrm{cm}$. Commercial SCL materials were kindly provided by Bausch \& Lomb as flat, rectangular sheets $(7 \times 9.5 \mathrm{~cm})$ synthesized between two glass plates. Two soft-contact-lens (SCL) materials were used in this study: a conventional hydrogel, SofLens ${ }^{\mathrm{TM}}$ One Day (hilafilcon A), and a last-generation silicone-hydrogel, PureVision $^{\mathrm{TM}}$ (balafilcon A). One batch of PureVision ${ }^{\mathrm{TM}}$ sheets was subjected to surface oxygen plasma treatment, similar to the commercial product [36-38], whereas a second batch was not.

Table 1 gives properties of SCL membranes, including a previously studied HEMA (2-hydroxyethyl methacrylate) SCL [29]. Water content at saturation, $w_{10}$, reported by Bausch \& Lomb, refers to weight percent of water in a wet contact lens that has been completely water saturated in a sterile isotonic solution at room temperature. The conventional hydrogel SofLens ${ }^{\mathrm{TM}}$ One Day has a water content at saturation of $70 \%$, much larger than that of HEMA at $38 \%$. The saturation water content of PureVision ${ }^{\mathrm{TM}}$ is $36 \%$, similar to that of HEMA.

Wet thickness, $L_{0}$, of the SCL membranes was measured using a micrometer

(Mitutoyo MDC 0-1" PF, accuracy $\pm 1 \mu \mathrm{m}$ ) at different locations; the average value reported in Table 1 (with the standard deviation) is used for subsequent calculations. 
From the small standard deviation, we conclude that the lenses have an essentially uniform thickness. The glass-transition temperature of each dry SCL material was measured by modulated differential scanning calorimetry (Modulated DSC 2920, TA Instruments) as described elsewhere [39]. Table 1 also reports the dry mass density, $\rho_{p}$, for each contact lens, as estimated from the water content and from the specific gravity of the saturated SCL (reported by Bausch \& Lomb) by assuming ideal volume of mixing. Flory interaction parameters, $\chi$, are calculated as discussed later.

\section{Apparatus}

The right side of Fig. 1 shows a schematic of the evaporation cell used to measure diffusivities of water through soft-contact-lens membranes [28]. The SCL membrane supports a small reservoir of water ( $10 \mathrm{~g})$ on the top surface while the bottom surface is exposed to impinging-air jet flow at fixed relative humidity $(R H)$ and flow rate. The soestablished chemical-potential gradient for water between the two membrane surfaces (i.e. the water content difference) induces an evaporative water flux across the lens that is measured gravimetrically. The evaporation cell, which sits atop an electronic balance (Sartorius, Model CP 343 S) is similar to that of Hoch et al. [28]. A detailed description of the modifications may be found in the thesis of Fornasiero [40].

A brass stand pipe is located below the membrane surface to provide an air supply of known humidity. The ratio between the pipe diameter and the SCL-exposed diameter $(2.29 \mathrm{~cm})$ is chosen to provide uniformly accessible mass transfer across the membrane surface [41]. Turbulent air flow is desired because measured water evaporation fluxes at finite air-flow Reynolds numbers, $R e$, must be extrapolated to infinite $R e$ (see next section). Large air-flowrates are required to reach the turbulent regime in an empty pipe. 
Further, when directly blown against the SCL membrane, such high flow rates cause damage and a large, lift-induced scattering of weight measurements. To overcome these difficulties, a 12 -element Kenics ${ }^{\circledR}$ static mixer is inserted into the brass air-supply stand pipe. The Kenics ${ }^{\circledR}$ static mixer produces turbulent flow at $R e$ as low as 500 (supplier specifications and [42]). As illustrated on the left of Fig. 1, dry, compressed air (relative humidity $R H=5-7 \%$ ) from the laboratory line is first filtered to remove dust and oil droplets. The air supply is then split into two lines. One line feeds the dry air to a humidification column $(H=100 \mathrm{~cm}$; OD $=10 \mathrm{~cm})$ where it is water saturated by countercurrent contact with a water stream in a packed column (Berl saddles, nominal size $1 / 2$ in, 40-cm height). The other line bypasses the humidification column and is recombined with the wet-air line in a ratio required to reach the desired $R H$.

A mass-flow sensor (Sensirion, Model ASF 1400) and a humidity/temperature sensor (Sensirion, Model SH 11) allow real-time monitoring of the total air flow rate, temperature, and relative humidity. Together with the electronic balance, they are interfaced to a computer through a dedicated LabView ${ }^{\circledR}$ program for automatic data collection.

Before measuring steady-state water fluxes through the membranes, we verified that no other water loss occurs in the evaporation cell. Instead of a SCL membrane, we replaced the lens material with an impermeable aluminum foil in the evaporation cell and measured the weight loss as in a typical water-flux experiment. The measured water flux was less than $1 \%$ of the lowest observed flux with a SCL material in place. We conclude that our evaporation cell is tightly sealed. 


\section{Method}

SCL membranes are removed from the original ReNu Multiplus ${ }^{\circledR}$ (Bausch \& Lomb) disinfecting solution and rinsed thoroughly with distilled/deionized water. Then, the membranes are soaked in distilled/deionized water for at least three days. Water is renewed every $24 \mathrm{~h}$ to ensure complete removal of the disinfecting solution. Upon completion of the cleaning procedure, the membranes are immersed in fresh distilled/deionized water and stored in a refrigerator to prevent bacterial growth.

After choosing the specific type of lens to be studied, a sheet of the contact-lens material is sandwiched between two supporting Delrin ${ }^{\circledR}$ rings, and $10 \mathrm{~g}$ of distilled/deionized water are placed on the top surface of the sandwiched membrane. Two layers of Saran ${ }^{\mathrm{TM}}$ wrap are then fastened over the top ring, such that the water-containing chamber is sealed and undesired evaporation from the top surface is minimized. The evaporation cell is then placed on the balance. Once the desired air flow rate and relative humidity are attained, measurements of mass, flow rate, relative humidity, temperature and time are recorded every $15 \mathrm{~s}$ for at least $4 \mathrm{~h}$ with a LabView ${ }^{\circledR}$ program. Water massloss measurements are averages over this 15 -s time interval.

Steady-state conditions are reached when the water flux through the lens is constant, as verified by comparing the slope of mass-versus-time data at various intervals of time. The last-hour slope is used to calculate the steady-state water mass flux, $n_{l}$. Typically, $n_{l}$ differs by less than $1 \%$ from the last two-hours slope.

For each lens material, experimental data are collected for seven different air-flow rates in the range $6-50 \mathrm{~L} / \mathrm{min}$ and for three different relative humidities at $24.0 \pm 0.5^{\circ} \mathrm{C}$. Chosen air-flow rates are $6,8,10,14,20,30$ and $50 \mathrm{~L} / \mathrm{min}$, while relative humidities are 
20,50 , and $80 \%$. Air flow and relative humidity are maintained constant within $\pm 0.5 \%$ and $1 \%$ of the set points, respectively. Each experiment is repeated at least twice to assure data reproducibility. Repeat experiments yield water fluxes that typically agree to within $2-4 \%$. Additional details on the experimental apparatus and measurement procedures are available elsewhere [40].

In general, a mass-transfer resistance for water is present at the membrane/air interface. This interfacial resistance is in series with the diffusive resistance of the membrane and must be accounted for. For impinging-jet flow, the mass-transfer coefficient scales with the square root of the air-flow rate [41]. Therefore, a plot of $1 / n_{1}$ as a function of $R e^{-1 / 2}$ should be linear. The intercept on the ordinate of this plot gives the inverse water flux for an infinite air-flow Re, i.e. in the absence of mass transfer resistance at the membrane/air interface [28]. This extrapolated water flux, $n_{1}^{\infty}$, is used here to determine the water diffusivity in the SCL material. Because of excellent reproducibility of measured fluxes, the experimental error for extrapolated water fluxes is small, as demonstrated in column 3 of Table 2.

\section{Extended Maxwell-Stefan Framework}

To interpret the water-evaporative flux results, we use the recently proposed extended Maxwell-Stefan (EMS) model [35]. Because the water head in the evaporationcell reservoir is very small, we assume that our system is isobaric. Further, we assume isothermal diffusion with no volume change upon mixing. Water transport occurs essentially only in the direction orthogonal to the membrane surface. Under these conditions, EMS reduces to 


$$
c_{1} \frac{d \mu_{1}}{d x}=R T \frac{\phi_{1} \phi_{p}}{v \bigoplus_{l p}}\left(\mathrm{v}_{p}-\mathrm{v}_{1}\right)
$$

where subscripts 1 and $\mathrm{p}$ denote water and polymer, respectively; $\mu_{1}$ is the local chemical potential of water in the membrane; $x$ is the distance from the upper surface of the membrane; $c_{1}$ is molar concentration of water in the water/polymer mixture; $\phi_{i}$ is volume fraction of $i ; \mathrm{v}_{i}$ is species $i$ velocity; $v$ is the polymer segment molar volume; and $\bigoplus_{l p}$ is the binary EMS diffusion coefficient for water in the membrane.

Since EMS regards a membrane/solvent system as a nonideal polymer/solvent mixture, the chemical potential of water in the membrane is calculated from the polymergel/solution theory of Flory and Rehner [28, 43-45]:

$$
\mu_{1}=\mu_{1}^{0}+R T\left[\ln \phi_{1}+\phi_{p}+\chi \phi_{p}^{2}+\frac{v_{1}^{0} G}{R T}\left(\phi_{p}^{1 / 3}-\frac{1}{2} \phi_{p}\right)\right]
$$

where the superscript 0 denotes a pure species, $\chi$ is the Flory solvent-polymer interaction parameter, $v_{1}^{0}$ is the molar volume of pure water, and $G$ is the membrane bulk modulus. The first three terms within the square brackets of Eq (2) correspond to FloryHuggins [46] theory for a polymer with an infinite molecular weight, whereas the last term provides the contribution of membrane elasticity. Here, the Flory interaction parameter $\chi$ is assumed constant and is obtained from the measured membrane water content when equilibrated in a sterile isotonic solution [28], as reported in Table 1 (the difference by water contents in distilled/deionized water and isotonic solution is minimal for the nonionic lenses studied). Precise values of $G$ for all SCL's are not available. Fortunately, calculations are not sensitive to the value of $G$. In our calculations, we use $G=1.0 \mathrm{MPa}$ for all SCL materials, close to that reported for PureVision ${ }^{\mathrm{TM}}[36,38,47]$. 
Appendix I gives calculation details for fitting EMS diffusivities $\bigoplus_{l p}$ while accounting for shrinking/swelling of the SCL membranes, and lists the boundary conditions for integration of Eq (1). For completeness, Appendix I also demonstrates the calculation of Fickian diffusion coefficients, $D_{l p}$, for our water/SCL membrane systems.

\section{Results}

In Figs. 2-5, we plot the inverse of the measured steady-state mass fluxes of water $1 / n_{1}$ as a function of air-flow $R e^{-1 / 2}$ for our contact-lens materials. Here, the reference diameter for $R e$ is that of the empty air-supply line. Best linear fits are also drawn for comparison. Clearly, the experimental data are well represented by fitting straight lines for all SCL membranes and for all relative humidities, indicating that our experiment obeys the impinging-jet flow conditions. Therefore, we can confidently extrapolate our measured water fluxes to infinite air-flow rate to obtain the steady-state diffusive mass fluxes $n_{1}^{\infty}$ through the SCL membranes in the absence of any external mass-transfer resistance. In these plots, the nonzero slope of the straight lines shows that the external mass transfer resistance cannot be neglected. This slope increases with rising air relative humidity in agreement with theoretical predictions [48].

Figs. 4 and 5 show measured steady-state fluxes for non-treated (NT) and surfacetreated (ST) PureVision ${ }^{\mathrm{TM}}$ lenses, respectively. $R H=50$ and $80 \%$ results for STPureVision $^{\mathrm{TM}}$ are almost indistinguishable from those for NT-PureVision ${ }^{\mathrm{TM}}$. Because the two materials present identical water fluxes at these $R H s$, we did not repeat the experiments for ST-PureVision ${ }^{\mathrm{TM}}$ to verify data reproducibility after investigating NTPureVision $^{\mathrm{TM}}$. At $R H=20 \%$, water fluxes for ST-PureVision ${ }^{\mathrm{TM}}$ are $10 \%$ lower than those for NT-PureVision ${ }^{\mathrm{TM}}$ at the same air-flow rate. Extrapolated water fluxes for ST- 
PureVision $^{\mathrm{TM}}$ differ less than $10 \%$ from those for NT-PureVision ${ }^{\mathrm{TM}}$ at the same $R H$ (see Table 2). The oxygen-plasma surface treatment creates small, hydrophilic silicate islands on the surface of ST-PureVision ${ }^{\text {TM }}$ contact lens $[37,38,49]$. These islands are separated by the nascent, hydrophobic silicone-hydrogel material (balafilcon-A), and are elevated by about $10-50 \mathrm{~nm}$ [36-38]. Because only a partial area of the lens surface is affected by plasma treatment and only for a shallow thickness, material bulk properties such as diffusivity are expected to be, at most, marginally affected by surface treatment, consistent with our experimental findings. Because ST-PureVision ${ }^{\mathrm{TM}}$ and NTPureVision ${ }^{\mathrm{TM}}$ contact-lens materials have essentially the same equilibrium and transport properties for water, we refer to these materials simply as PureVision ${ }^{\mathrm{TM}}$ in the remaining discussion.

Table 2 lists the extrapolated fluxes $n_{1}^{\infty}$. In this table columns 2 and 3 reported measured quantities, whereas column 4-9 list calculated values from EMS or Fick's laws, as described in Appendix I. Comparison of $n_{1}^{\infty}$ at the same relative humidity for HEMA and SofLens ${ }^{\mathrm{TM}}$ One Day materials shows that the water flux is significantly larger, by an order of magnitude, for the high-water-content SofLens ${ }^{\mathrm{TM}}$ One Day lens. Similarly, Martin [8] reported a positive correlation between steady-state evaporative loss and water content for conventional soft-contact-lens materials. This result is also consistent with clinical and experimental observations that conventional hydrogel contact lenses with higher water content at saturation tend to dehydrate on eye more quickly and in a greater amount $[1,5,50,51]$. Silicone-hydrogel PureVision ${ }^{\mathrm{TM}}$ has a water content at saturation that is slightly lower than that for HEMA. However, the measured water fluxes are twice as large. To our best knowledge, there are no previous published data for water fluxes 
through silicone hydrogel materials driven by a chemical-potential gradient. Some data have been reported for tritium-labeled water diffusion rates, which show a somewhat $(10 \%)$ higher water transport for PureVision ${ }^{\mathrm{TM}}$ compared to that in HEMA $[16,36]$.

\section{Discussion}

The calculated steady-state membrane thickness for SofLens ${ }^{\mathrm{TM}}$ One Day is nearly half of those for HEMA and PureVision ${ }^{\mathrm{TM}}$ lens materials (see Tables 1 and 2), whereas the steady-state water fluxes for SofLens ${ }^{\mathrm{TM}}$ One Day are 10 and 5 times larger than those for HEMA and PureVision ${ }^{\mathrm{TM}}$, respectively. Thus, the large variation in water fluxes must be primarily related to the physico-chemical properties of the lens membranes. SofLens ${ }^{\mathrm{TM}}$ One Day is a copolymer of HEMA and N-vinyl-pyrrolidone (VP). The latter monomer confers augmented hydrophilicity to the SCL material, increasing saturation water content from 38 (HEMA homopolymer) to $70 \%$. Moreover, polyvinylpyrrolidone exhibits water diffusivities greater than those in HEMA [52-55]. Therefore, VP enhances water transport in SofLens ${ }^{\mathrm{TM}}$ One Day by increasing both water uptake and diffusivity in the SCL material. PureVision ${ }^{\mathrm{TM}}$ has a more complex chemical composition with the exact formulation proprietary. However, literature indicates that PureVision ${ }^{\mathrm{TM}}$ is a copolymer of tris-(trimethylsiloxy)-silyl-propylvinyl carbamate (TRIS-VC), N-vinylpyrrolidone [56], a vinyl carbonate functional polydimethylsiloxane (PDMS) macromer, and a vinyl carbamate derivative of alanine [47]. Although water uptake for PureVision $^{\mathrm{TM}}$ is essentially the same as that for HEMA, water diffusivity is most likely augmented by the presence of both silicone $[57,58]$ and VP groups.

To interpret our experimental fluxes with EMS or Fick's law, we first take the EMS $\left(\bigoplus_{l_{p}}\right)$ or Fickian $\left(D_{l p}\right)$ diffusivities as concentration independent, and we fit $\bigoplus_{l_{p}}$ or 
$D_{l p}$ separately for each $R H$ condition, as explained in Appendix I. Table 2 lists the resulting diffusivities, average steady-state water-content $\left(<w_{1}>\right)$ and fractional thickness variation calculated both with the EMS model and with Fick's law. At fixed $R H$, the largest diffusivity is obtained for SofLens ${ }^{\mathrm{TM}}$ One Day followed by PureVision ${ }^{\mathrm{TM}}$ and HEMA. This order is consistent with the previous analysis based on membrane chemistry. Clearly, however, the values of $\bigoplus_{l p}$ and $D_{l p}$ both increase significantly with average water content for all SCL membranes. The starting assumption of concentrationindependent diffusivities is, therefore, not satisfied.

\section{Concentration dependent diffusivities}

In an attempt to account for the strong water-content dependence of the diffusivities, we assumed an exponential variation with water volume fraction, $\bigoplus_{l p}=a \exp \left(b \phi_{1}\right)$, where $a$ and $b$ are constants; we fit the measured water fluxes to obtain parameters $a$ and $b$ according to the procedure outlined in Appendix I. Unfortunately, fitting results were poor. To resolve this discrepancy, we first questioned the assumption of isothermal diffusion as outlined in Appendix II. Our calculations show that evaporative cooling at the membrane/air interface has little impact on the measured water fluxes (see Table 3). Hence, our systems can be effectively considered isothermal.

To explore further the reasons for our inability to describe the $R H$ dependence of our extrapolated water fluxes, $n_{1}^{\infty}$, we examined the trend of measured water fluxes as a function of $R H$. Because a higher air $R H$ corresponds to a lower driving force for water flux, one expects lower fluxes at higher $R H$. For HEMA $n_{1}^{\infty}$ indeed decreases (or remains unchanged within experimental uncertainty) with increasing $R H$. However, the opposite 
trend and a minimum are observed with SofLens ${ }^{\mathrm{TM}}$ One Day and PureVision ${ }^{\mathrm{TM}}$ materials, respectively. Crank [59, 60] reports similar increases in steady-state diffusive transport for increasing gas concentration of an evaporating solvent from polymer membranes. Such increase of solvent flux with a smaller driving force is commonly observed in drying technology and is termed "trapping skinning" [61].

In EMS, the water flux at steady state can be written in the form

$$
n_{1}^{\infty}=\frac{1}{L} \int_{\phi_{1 L}}^{\phi_{10}} \frac{\bigoplus_{l p}}{R T} \frac{\phi_{1}}{\phi_{p} \hat{v}_{1}^{0}} \frac{d \mu_{1}}{d \phi_{1}} d \phi_{1} .
$$

where $\hat{v}_{1}^{0}$ is the specific volume of pure solvent. The derivative of the chemical potential with composition is positive for a stable mixture. For any non-negative concentrationdependent $\bigoplus_{l p}$, the integrand in Eq (3) is non-negative. Since the upper limit of integration in Eq (3) is fixed and the lower limit, $\phi_{1 L}$, decreases by lowering the air $R H$, EMS predicts a steady-state flux that always increases when the air $R H$ decreases. Therefore, our model cannot predict trapping skinning for any $\bigoplus_{l p}$ that is a non-negative function of concentration. Arguments presented in Appendix III show that accounting for shrinking/swelling does not relieve the discrepancy in the EMS model. Note, also, that the same result is encountered in Fickian diffusion models [60, 62].

Although trapping skinning was first observed several decades ago, there is no clear physical understanding of this phenomenon. It has been hypothesized [61, 63-65] that trapping skinning may occur when the surface of the polymeric material exposed to the atmosphere undergoes a rubber-to-glass transition during drying. A recent study using atomic force microscopy $[66,67]$ showed that such transition occurs in some HEMAbased SCLs when environmental relative humidity drops below 50-60\%. We have also 
investigated the possibility of glassy-skin formation for our SCL materials by measuring glass transition temperatures as a function of water content with modulated differential scanning calorimetry [39]. We find that, for the formation of a glassy skin, a local water content is required of less than 12,15 , and 7 weight $\%$ for HEMA, SofLens ${ }^{\mathrm{TM}}$ one day and PureVision ${ }^{\mathrm{TM}}$ materials, respectively. The corresponding air $R H$ ( $\sim$ water activity) can be estimated from equilibrium water-activity versus water-content data obtained with a gravimetric sorption technique at $35{ }^{\circ} \mathrm{C}$ [68]. Our results indicate that the rubber-toglass transition occurs at estimated $R H$ of $74 \%, 64 \%$ and $57 \%$ for HEMA, SofLens ${ }^{\mathrm{TM}}$ One Day and PureVision ${ }^{\mathrm{TM}}$, respectively. Therefore, it is likely that all three SCL materials exhibit a glassy skin at the membrane/air interface when the experimental $R H$ is equal to $20 \%$ and $50 \%$ in Table 2 , which may explain the existence of trapping skinning. For HEMA, the appearance of a glassy skin apparently compensates exactly the effect of increased driving force for water flux when the $R H$ is reduced from $50 \%$ to $20 \%$. For HEMA, no substantial variation with $R H$ is observed in the measured water fluxes.

For SofLens ${ }^{\mathrm{TM}}$ One Day material, however, trapping skinning overpower the chemical potential gradient driving water transport leading to a decreasing water flux with decreasing $R H$. Therefore, consistent with on our bulk $T_{g}$ measurements and with the surface $T_{g}$ experiments of Opdahl et al. [66] and Koffas et al. [67], trapping skinning is the most likely explanation for the unsuccessful fitting of our experimental fluxes with a diffusivity increasing with membrane water-concentration. A concentration-dependent diffusivity cannot explain the water flux data for the studied contact lens materials.

Only a few models $[61,63-65]$ have been proposed to explain trapping skinning, mainly for the drying of polymeric coatings. One model invokes a viscoelastic diffusion 
process for a polymer/solvent mixture near its glass-to-rubber transition temperature [61, 63, 64]; another model assigns its capability of predicting trapping skinning to incorporation of a non-ideal volume change in the glassy region of the polymer/solvent mixture [65]. Unfortunately, we cannot directly apply these models to our systems because many of the required parameters are not available or cannot be easily obtained for our SCL materials. However, we believe that it is unlikely that the incorporation of a non-ideal volume change can explain our results. Indeed, Appendix III shows that the steady-state water flux must increase upon reduction of external $R H$, except in the unlikely instance that the partial specific volume for the SCL polymer is negative. We conclude that accounting for non-ideal volume of mixing in a glassy skin cannot represent trapping skinning in our systems.

Other models may be proposed to represent trapping skinning. For example, we may consider a surface resistance on the SCL - surface exposed to air which decreases upon raising the external relative humidity. However, the number of parameters required to fit our extrapolated water fluxes is too large to obtain unambiguous values. Because EMS (and Fick's law) do not account for a rubber-to-glass transition and cannot represent trapping skinning, we can only report the concentration-independent diffusivities listed in Table 2; these reported diffusivities must be considered as effective diffusivities when the $R H$ is below the critical $R H$ for the glass-to-rubber transition.

\section{Soft contact lens wear}

At the beginning of a blink cycle, a soft contact lens on-eye is normally bathed in the tear film. The pre-lens tear film (PrLTF) on a soft contact lens is generally unstable and ruptures several seconds after lid opening [69-71]. Some regions of the SCL surface 
are then exposed directly to external air [72]. Upon PrLTF rupture, an evaporativedehydration process commences that draws water through the contact lens and out of the post-lens tear film toward the environment [2-7]. A subsequent blink deposits a new PrLTF on the SCL surface, and the contact lens partially rehydrates. This process repeats cyclically at each blink. After some time, the soft contact lens reaches a periodic steady state $[2,48]$. In this condition, the SCL has constant water content when averaged over a blink cycle. However, there remains a non-zero water flux from the PoLTF through the contact lens toward the external air. The amount and the rate of dehydration depend on a large number of factors including water diffusivity in the SCL material, SCL thickness, PrLTF stability, SCL surface area exposed to air upon PrLTF rupture, blink rate, external $R H$, and wind velocity. Because water diffusivity and water content are higher for SofLens $^{\mathrm{TM}}$ One Day, we expect SofLens ${ }^{\mathrm{TM}}$ One Day to be more prone to in-vivo dehydration and PoLTF collapse than HEMA and PureVision ${ }^{\mathrm{TM}}$ contact lenses. From this point of view, high water transport rates through SCLs are undesirable. Consequently, formation of a glassy skin and a water diffusivity that increases with water concentration may be beneficial for a soft contact lens worn on the eye because they limit lens dehydration in low humidity environments. Conversely, physical interaction of the eyelid with a glassy skin may lead to discomfort [73].

\section{Conclusions}

We have investigated water transport through typical soft-contact-lens (SCL) materials with an evaporation-cell method. Measured steady-state water fluxes are largest for SofLens ${ }^{\mathrm{TM}}$ One Day $\left(\sim 50-70 \times 10^{-6} \mathrm{~g} \mathrm{~cm}^{-2} \mathrm{~s}^{-1}\right)$, an order of magnitude greater than those for HEMA membranes $\left(\sim 4-5 \times 10^{-6} \mathrm{~g} \mathrm{~cm}^{-2} \mathrm{~s}^{-1}\right)$. For PureVision ${ }^{\mathrm{TM}}$, the surface 
oxygen-plasma treatment does not affect water transport through the soft contact lens. For SofLens ${ }^{\mathrm{TM}}$ One Day and (in some cases) for PureVision ${ }^{\mathrm{TM}}$ materials, water fluxes increase with rising environmental relative humidity. This phenomenon is associated with formation of a glassy skin at the membrane/air interface. Independent measurements of the glass-transition temperature for these SCL-polymers as a function of water content strongly suggest that our SCL materials develop a glassy skin when $R H=20 \%$ and $R H=$ $50 \%$, whereas our lenses remain rubbery at $R H=80 \%$.

Steady-state water fluxes are interpreted with an extended Maxwell-Stefan (EMS) model for a homogeneous mixture of molecules of starkly different size. Fick's law diffusion coefficients are also reported. Fitted (effective) diffusivities increase with rising

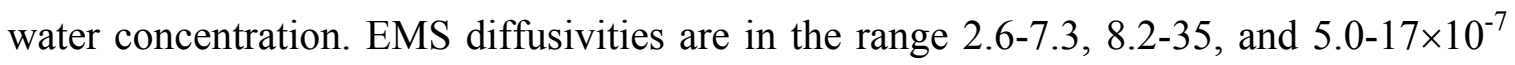
$\mathrm{cm}^{2} \mathrm{~s}^{-1}$, respectively, for HEMA, SofLens ${ }^{\mathrm{TM}}$ One Day and PureVision ${ }^{\mathrm{TM}}$; corresponding Fickian diffusivities are in the range 1.7-3.4, 5.3-16 and 3.3-8.4 $\times 10^{-7} \mathrm{~cm}^{2} \mathrm{~s}^{-1}$. The faster water transport and higher water content of SofLens ${ }^{\mathrm{TM}}$ One Day suggest that this lens material is more prone to in-vivo dehydration and PoLTF depletion than HEMA and PureVision $^{\mathrm{TM}}$.

\section{Acknowledgements}

For financial support, the authors are grateful to the Office for Basic Sciences of the U. S. Department of Energy and to the Donors of the Petroleum Research Fund, administered by the American Chemical Society; to G. Friends of Bausch \& Lomb for kindly providing soft-contact-lens materials; to I H. Romdhane for helpful comments, and to I. Loy, F. Sim, N. Dutta, A. Sutarman and T. T. Cheong for help with the 
experimental work. F. Krull thanks the "Prof. Dr. Ing. Erich Mueller Stiftung" and the "Rheinstahlstiftung" (Germany) for financial support. 


\section{Appendix I. Fitting Diffusivities to Evaporation-Cell Water Fluxes: Mathematical Details}

Eq (1) in the text gives the EMS diffusion equation for an isothermal, isobaric membrane/water system. At steady state, the membrane polymer species velocity is zero and the water velocity (or flux) is a constant. Because the chemical potential in FloryRehner theory is expressed as a function of volume fraction, it is convenient to rewrite Eq (1) as

$$
\frac{d \mu_{1}}{d \ln \phi_{1}} \frac{d \phi_{1}}{d x}=-\frac{R T}{\bigoplus_{l p}} \phi_{p} \hat{v}_{1}^{0} n_{1}^{\infty}
$$

where $n_{1}^{\infty}$ is the extrapolated mass water flux through the SCL material at steady-state. Eq (I.1) is a first-order nonlinear differential equation with an unknown parameter, the diffusivity $\bigoplus_{l p}$. We want to obtain $\bigoplus_{l p}$ by fitting to our measured water fluxes, $n_{1}^{\infty}$, at steady state.

Unfortunately, integration of Eq (I.1) is not straightforward since the steady-state thickness $L$ of the membrane is unknown because the membrane swells or shrinks in response to the water-content profile. To account self-consistently for swelling/shrinking of the membrane, we impose conservation of the total polymer mass [28, 74]. Thus, we define a variable $\eta$ representing the mass of polymer per unit area between $x=0$ and $x$,

$$
\eta(x)=\int_{0}^{x} \frac{\phi_{p}}{\hat{v}_{p}} d x
$$

where $\hat{v}_{p}$ is the partial specific volume for polymer. Polymer mass conservation requires that $\eta(L)$ is a constant $\left(=L_{0} \phi_{p 0} / \hat{v}_{p}\right)$. It is convenient to write Eq (I.2) in differential form and to solve it simultaneously with Eq (I.1) [74]. We define a dimensionless 
distance $\xi=x / L$. After replacing $x$ with $\xi$ in Eq (I.2), we reduce the integral equation to a first-order differential equation or

$$
\frac{d \eta}{d \xi}=L \frac{\phi_{p}}{\hat{v}_{p}}
$$

To now solve for the membrane thickness we note that $L$ is a scalar independent of position so that

$$
\frac{d L}{d \xi}=0
$$

In general, the EMS diffusivity is a function of composition. Since this dependence is not known, we first assume that $\bigoplus_{l p}$ constant and then, if needed, relax this constraint. We express the constancy of $\bigoplus_{1 p}$ as

$$
\frac{d \bigoplus_{l p}}{d \xi}=0
$$

After rewriting $\mathrm{Eq}$ (I.1) in terms of the variable $\xi$, we solve numerically the set of coupled, nonlinear, first-order ordinary differential Eqs (I.1), and (I.3)-(I.5).

\section{Boundary Conditions}

Four boundary conditions are required. Since there is no mass-transfer resistance at the air/membrane interface at infinite air-flow rate, the volume fraction of water at the bottom surface of the membrane, $\phi_{1 L}$, is set by thermodynamic equilibrium with water vapor in air at the bulk relative humidity. Thus, the boundary condition at the membrane surface exposed to air is

$$
\phi_{1}=\phi_{1 L} \quad \text { at } x=L
$$


where $\phi_{1, L}$ is calculated as explained earlier [28]. The other surface of the membrane is in equilibrium with pure water. Therefore, we have that

$$
\phi_{1}=\phi_{10} \quad \text { at } x=0
$$

where $\phi_{10}$ is calculated from the membrane water content at saturation [28]. The associated boundary conditions for Eqs (I.3) and (I.4) are, respectively

$$
\begin{aligned}
& \eta(0)=0 \\
& \eta(1)=L_{0} \phi_{p 0} / \hat{v}_{p} .
\end{aligned}
$$

For each relative humidity, we solve simultaneously the set of coupled nonlinear differential equations using a finite-difference scheme with Newton iteration of the nonlinearities (subroutine BAND(J) [75]).

\section{EMS Diffusivities}

As shown in the Discussion section, the resulting $\bigoplus_{l p}$ values vary with relative humidity. In particular, $\bigoplus_{l p}$ increases significantly with the average water content in the lens. Therefore, we relax the hypothesis of constant $\bigoplus_{l p}$ and assume an exponential concentration dependence for $\bigoplus_{l p}$

$$
\bigoplus_{l p}=a \exp \left(b \phi_{1}\right)
$$

where $a$ and $b$ are constants. We use a Levenberg-Marquardt method [76] to fit these constants to the measured steady-state water fluxes in Table 2.

\section{Fickian Diffusivities}

Because most researchers report Fickian diffusivities for small penetrants in polymeric membranes, we calculate here the diffusion coefficient of Fick's law for our 
systems. Under the assumption of a concentration-independent $D_{l p}$, the Fickian waterconcentration profile in the membrane is linear at steady state. Steady-state thickness $L$ can be determined analytically by integration of Eq (I.2). Fickian diffusivities are then given by

$$
D_{1 p}=\frac{N_{1}^{\infty} L}{c_{10}-c_{1 L}}
$$

where $c$ indicates a molar concentration and $N_{1}^{\infty}$ is the extrapolated molar flux of water. We do not attempt to fit concentration-dependent Fickian diffusivities. 


\section{Appendix II. Impact of Evaporative Cooling on Steady-State Water Fluxes}

During an evaporation-cell experiment, cooling occurs on the bottom SCL surface because of water evaporation. We want to assess the importance of evaporative cooling in our evaporation-cell experiments by comparing calculated water steady-state fluxes for isothermal and nonisothermal diffusion. The non-isothermal case requires solving simultaneously heat transfer (HT) and mass transfer (MT) equations in a membrane/air system. At steady state, the equations and boundary conditions for HT and MT in the membrane are, respectively [77]:

$$
\begin{array}{lll}
\frac{d n_{1}}{d x}=0 & w_{1}(x=0)=w_{10}, & w_{1}(x=L)=w_{1 L} ; \\
\frac{d e}{d x}=0 & T(x=0)=T_{0}, & T(x=L)=T_{L},
\end{array}
$$

where

$$
n_{1}=\frac{\rho D_{1 p}}{1-w_{1}} \frac{d w_{1}}{d x}
$$

and

$$
e=-k_{T} \frac{d T}{d x}+n_{1} c_{p}\left(T-T_{0}\right)
$$

In Eqs (II.1)-(II.4), $T$ indicates temperature, and $w_{1}$ is the water weight fraction; subscripts 0 and $L$ indicate membrane/water and membrane/air interfaces, respectively; $k_{T}$ is the thermal conductivity for the water/membrane mixture; $c_{p}$ is the specific heat capacity for liquid water; $\rho$ is the wet-SCL mass density; $n_{1}$ is the water steady mass flux, and $e$ is a combined energy flux defined by equations (19.3-4) and (19.3-6) in reference [77]. To derive Eq (II.3), we used Fick's law to express the water diffusive 
flux. In Eq (II.4), the first term on the right represents heat conduction, while the second term corresponds to the enthalpy flux associated with mass transport [77].

The water weight fraction, $w_{1 L}$, and the temperature, $T_{L}$, at the membrane/air interface are unknown. We obtain these quantities by setting the fluxes $n_{1}$ and $e$ in the membrane equal to the corresponding fluxes on the airside of the membrane/air interface:

$$
\begin{aligned}
& n_{1}=k_{1} \rho_{\text {air }}\left(\frac{w_{1 L}^{\text {air }}-w_{1}^{\text {air }}}{1-w_{1 L}^{\text {air }}}\right) \\
& e=h_{T}\left(T_{L}-T_{0}\right)+n_{1} c_{p v}\left(T_{L}-T_{0}\right)+\lambda_{1} n_{1}
\end{aligned}
$$

where superscript air indicates air side; $k_{1}$ and $h_{T}$ are mass transfer and heat transfer coefficients at the SCL membrane/air interface, respectively; $\rho_{a i r}$ is air mass density; $c_{p v}$ is specific heat capacity for water vapor, and $\lambda_{1}$ is the enthalpy of vaporization for water. In Eq (II.6), the first term represents the heat flux in absence of simultaneous mass transfer, while the second and third terms give the heat flux associated with water-vapor transport and water vaporization, respectively. We assume that the temperature in bulk air is equal to that of the water reservoir $\left(T_{0}=25^{\circ} \mathrm{C}\right)$, and that $w_{1 L}^{\text {air }}$ and $w_{1 L}$ are related by local equilibrium.

To obtain $T_{L}$ and $w_{1 L}$, we use an iterative procedure. For the isothermal case, only Eq (II.5) is required, and every quantity appearing there is evaluated at temperature $T_{0}$. Details on parameter estimates and calculation procedure are given elsewhere [40].

Table 3 summarizes the results of calculations for coupled HT and MT equations. Table 3 shows that cooling at the membrane/air interface does not exceed $3-4{ }^{\circ} \mathrm{C}$, and that it is larger for smaller $R H$. Water flux is reduced by evaporative cooling. The effect is the 
greatest for SofLens ${ }^{\mathrm{TM}}$ One Day at the lowest $R H$, because the evaporative fluxes are the largest for this SCL material. Despite conservative assumptions for $D_{1 p}$ and $k_{T}$, water fluxes for nonisothermal diffusion differ little from those obtained in the isothermal case. These results indicate that evaporative cooling at the membrane/air interface is not important for interpreting our water flux measurements. 


\section{Appendix III. Predicted Dependence of Steady-State Water-Flux on External Relative Humidity for Fickian or Maxwell-Stefan Diffusion Models.}

According to a recently proposed drying model [65], trapping skinning in unsteady-state drying of polymer films can be explained by nonideal volumetric mixing behavior exhibited by a glassy polymer-solvent mixture. We show here that, under the assumption that the solvent diffusivity in a binary solvent/polymer system is a nonnegative function of concentration only, accounting for nonideal volumetric behavior is insufficient to capture trapping skinning in an isothermal permeation experiment at steady-state conditions.

As example, consider a membrane one surface of which is in equilibrium with pure water (located at $x=0$ ) and the other is in equilibrium with air at a $R H<100 \%$ (located at $x=L$ ), respectively. We investigate how the steady-state flux changes by varying the $R H$. The mass flux of solvent 1 is

$$
n_{1}=\rho_{1} \mathrm{v}_{1}=\rho_{1} \mathrm{v}^{\square}+j_{1}^{\square}
$$

where $\mathrm{v}^{\square}$ is the volume average velocity, $\mathrm{v}^{\square}=\rho_{1} \hat{v}_{1} \mathrm{v}_{1}+\rho_{2} \hat{v}_{2} \mathrm{v}_{2}, j_{1}^{\square}$ is the diffusive flux with respect to the volume average velocity, $\rho_{i}$ is a mass concentration and $\hat{v}_{i}$ is a specific partial volume for species $i$. The diffusive flux $j_{1}^{\square}$ can be expressed as a product of a non-negative diffusivity $D\left(\rho_{1}\right)$, a function of composition only, and a gradient of composition:

$$
j_{1}^{\square}=-D\left(\rho_{1}\right) \frac{d \rho_{1}}{d x}
$$


Because $D\left(\rho_{1}\right)$ can be any non-negative function of composition, Eq (III.2) includes Fick's law, EMS or the diffusion models used in reference [65]. At steady state, the polymer velocity is zero or

$$
n_{1}=j_{1}^{\square} /\left(1-\rho_{1} \hat{v}_{1}\right)=-\frac{D\left(\rho_{1}\right)}{\left(1-\rho_{1} \hat{v}_{1}\right)} \frac{d \rho_{1}}{d x},
$$

and the water flux, $n_{1}$, is constant. After multiplying both sides of Eq (III.3) by $\rho_{2} d x$, integration of Eq (III.3) results in

$$
n_{1}=\frac{1}{M} \int_{\rho_{1 L}}^{\rho_{10}} \frac{D\left(\rho_{1}\right)}{\hat{v}_{2}} d \rho_{1}
$$

where $\rho_{10}$ and $\rho_{1 L}$ are the solvent mass concentrations at the membrane surfaces at $x=0$ and $x=L,\left(1-\rho_{1} \hat{v}_{1}\right)=\rho_{2} \hat{v}_{2}$, and $M$ is the total polymer mass per unit area given by

$$
M=\int_{0}^{L} \rho_{2} d x .
$$

In Eq (III.4), $\rho_{10}$ is the solvent mass concentration in equilibrium with a pure solvent phase and $\rho_{1 L}$ is the solvent mass concentration in equilibrium with air at a given $R H$. Since the upper limit of integration is fixed and the lower limit decreases by lowering air $R H$, the range of integration rises when air $R H$ falls. For any non-negative concentrationdependent $D\left(\rho_{1}\right), n_{1}$ must increase by widening the range of integration, except when the partial specific volume of the polymer is negative. A negative $\hat{v}_{2}$ is unlikely. Therefore, except in the rare event of a negative $\hat{v}_{2}$, taking into account the non-ideal volumetric behavior in a glassy polymer-solvent mixture is not sufficient to explain a decreasing steady-state solvent flux when air $R H$ falls. 
Water Diffusion through SCLs

\section{References}

1. Andrasko G. Hydrogel Dehydration in Various Environment. ICLC $1983 ; 10(1): 23-28$.

2. Fatt I. A Predictive Model for Dehydration of a Hydrogel Contact Lens in the Eye. J Brit Contact Lens Assoc 1989;12(2):15-31.

3. Brennan NA, Efron N, Bruce AS, Duldig DI, Russo NJ. Dehydration of Hydrogel Lenses - Environmental-Influences During Normal Wear. Am J Optom Phys Opt $1988 ; 65(4): 277-281$.

4. Efron N, Brennan NA, O'Brien KA, Murphy PJ. Surface Hydration of Hydrogel Contact Lenses. Clin Exp Optom 1986;69(6):219-222.

5. Efron N, Brennan NA, Spruce AS, Duldig DI, Russo NJ. Dehydration of Hydrogel Lenses Under Normal Wearing Conditions. CLAO J 1987;13(3):152-156.

6. Refojo MF, Leong FL. Water pervaporation Through Silicone Rubber Contact Lenses: a Possible Cause of Complications. Contact Intraocular Lens Med J 1981;7(3):226-233.

7. Refojo MF. The Tear Film and Contact Lenses: the Effect of Water Evaporation from the Ocular Surface. In: Fisiologia del Film Lacrimale. Simposio del 64 Congresso della Societa' Oftalmologica Italiana; 1984; Rome; 1984. p. 189-197.

8. Martin DK. Water Transport in Dehydrating Hydrogel Contact-Lenses Implications For Corneal Desiccation. J Biomed Mater Res 1995;29(7):857-865.

9. Orsborn GN, Zantos SG. Corneal Dessication Staining With Thin High Water Content Contact Lenses. CLAO J 1988;14(2):81-85. 
10. Little SA, Bruce AS. Postlens Tear Film Morphology, Lens Movement and Symptoms in Hydrogel Lens Wearers. Ophthal Physl Opt 1994;14(1):65-69.

11. Little SA, Bruce AS. Environmental Influences on Hydrogel Lens Dehydration and the Post-Lens Tear Film. ICLC 1995;22:148-155.

12. Golding TR, Harris MG, Smith RC, Brennan NA. Soft Lens Movement - Effects of Humidity and Hypertonic Saline On Lens Settling. Acta Ophthalmol Scan 1995;73(2):139-144.

13. Fonn D, Situ P, Simpson T. Hydrogel Lens Dehydration and Subjective Comfort and Dryness Ratings in Symptomatic and Asymptomatic Contact Lens Wearers. Optometry Vision Sci 1999;76(10):700-704.

14. Efron N, Morgan PB. Hydrogel Contact Lens Dehydration and Oxygen Transmissibility. CLAO J 1999;25(3):148-151.

15. Little SA, Bruce AS. Osmotic Determinants of Postlens Tear Film Morphology and Hydrogel Lens Movement. Ophthal Physl Opt 1995;15(2):117-124.

16. Nicolson PC, Baron RC, Chabrecek P, Court J, Domschke A, Griesser HJ, et al. Extended Wear Ophthalmic Lens. USA patent 5760100. 1998.

17. McConville P, Whittaker MK, Pope JM. Water and Polymer Mobility in Hydrogel Biomaterials Quantified by H-1 NMR: A Simple Model Describing both T-1 and T-2 Relaxation. Macromolecules 2002;35(18):6961-6969.

18. Manetti C, Casciani L, Pescosolido N. Diffusive Contribution to Permeation of Hydrogel Contact Lenses: Theoretical Model and Experimental Evaluation by Nuclear Magnetic Resonance Techniques. Polymer 2002;43(1):87-92. 
19. McConville P, Pope JM. H-1 NMR T-2 Relaxation in Contact Lens Hydrogels as a Probe of Water Mobility. Polymer 2001;42(8):3559-3568.

20. McConville P, Pope JM. A Comparison of Water Binding and Mobility in Contact Lens Hydrogels from NMR Measurements of the Water Self-Diffusion Coefficient. Polymer 2000;41(26):9081-9088.

21. Barbieri R, Quaglia M, Delfini M, Brosio E. Investigation of Water Dynamic Behaviour in Poly(HEMA) and Poly(HEMA-co-DHPMA) Hydrogels by Proton T-2 Relaxation Time and Self-Diffusion Coefficient NMR Measurements. Polymer 1998;39(5):1059-1066.

22. Peschier LJC, Bouwstra JA, Debleyser J, Junginger HE, Leyte JC. Water Mobility and Structure in Poly[2-Hydroxyethylmethacrylate] Hydrogels by Means of the PulsedField Gradient Nmr Technique. Biomaterials 1993;14(12):945-952.

23. Arce A, Fornasiero F, Rodriguez O, Radke CJ, Prausnitz JM. Sorption and Transport of Water Vapor in Thin Polymer Films at $35^{\circ} \mathrm{C}$. PCCP 2004;6(1):103-108.

24. Gates G, Harmon JP, Ors J, Benz P. 2,3-Dihydroxypropyl Methacrylate and 2Hydroxyethyl Methacrylate Hydrogels: Gel Structure and Transport Properties. Polymer $2003 ; 44(1): 215-222$.

25. Thimmegowda MC, Sathyanarayana PM, Shariff G, Ashalatha MB, Ramani R, Ranganathaiah C. Water Sorption Studies in a RGP Contact Lens Polymer Paraperm by Positron Lifetime Technique. Physica Status Solidi A-Appl Res 2002;193(2):257-270.

26. Sun YM, Lee HL. Sorption/Desorption Properties of Water Vapour in Poly(2Hydroxyethyl Methacrylate).1.Experimental and Preliminary Analysis. Polymer 1996;37(17):3915-3919. 
27. Sun YM. Sorption/Desorption Properties of Water Vapour in Poly(2Hydroxyethyl Methacrylate).2.Two-Stage Sorption Models. Polymer 1996;37(17):39213928.

28. Hoch G, Chauhan A, Radke CJ. Permeability and Diffusivity for Water Transport through Hydrogel Membranes. J Membrane Sci 2003;214(2):199-209.

29. Fornasiero F, Krull F, Prausnitz JM, Radke CJ. Diffusion of Water through a HEMA-based Soft Contact Lens. Fluid Phase Equilibr, in press 2004.

30. Wijmans JG, Baker RW. The Solution-Diffusion Model - a Review. J Membrane Sci 1995;107(1-2):1-21.

31. Fatt I, Goldstick TK. Dynamics of Water Transport in Swelling Membranes. J Colloid Sci 1965;20(9):962-989.

32. Monticelli MV, Chauhan A, Radke CJ. The Effect of Water Hydraulic Permeability on the Settling of a Soft Contact Lens on the Eye. Current Eye Research, in press 2004 .

33. Refojo MF. Permeation of Water through Some Hydrogels. J Appl Polym Sci 1965;9(10):3417-3426 .

34. Taylor R, Krishna R. Multicomponent Mass Transfer. New York: John Wiley \& Sons, Inc.; 1993.

35. Fornasiero F, Prausnitz JM, Radke CJ. Diffusion in Highly Asymmetric Systems. An Extended Maxwell-Stefan Model for Starkly Different-Sized, Segment-Accessible Chain Molecules. Macromolecules, in print 2004. 
36. Tighe B. Silicone Hydrogel Materials: How do They Work? In: Sweeney D, editor. Silicone Hydrogels: The Rebirth of Continuous Wear Contact Lenses. Oxford: Butterworth-Heinemann; 2000. p. 1-21.

37. Grobe G. Surface Engineering Aspects of Silicone-Hydrogel Lenses. Contact Lens Spectrum 1999;14(8):14s-17s supplement.

38. Grobe G, Kunzler JF, Seelye D, Salamone JC. Silicone Hydrogels for Contact Lens Application. In: Polym Mat Sci Eng; 1999; Anaheim, CA: ACS; 1999. p. 108-109.

39. Fornasiero F, Ung M, Radke CJ, Prausnitz JM. Glass Transition Temperature for Soft-Contact-Lens Materials. Dependence on Water Content. submitted to Polymer 2004. 40. Fornasiero F. Ph.D. Dissertation. Berkeley: University of California; in progress. 41. Chin D-T, Tsang C-H. Mass Transfer to an Impinging Jet Electrode. J Electrochem Soc 1978;125:1461-1470.

42. Fang JZ, Lee DJ. Micromixing Efficiency in Static Mixer. Chem Eng Sci 2001;56(12):3797-3802.

43. Flory PJ, Rehner J. Statistical Mechanics of Cross-Linked Polymer Networks. I. Rubberlike Elasticity. J Chem Phys 1943;11(11):512-520.

44. Flory PJ. Statistical Mechanics of Swelling of Network Structures. J Chem Phys 1950;18(1):108-111.

45. Flory PJ, Rehner J. Statistical Mechanics of Cross-Linked Polymer Networks. II. Swelling. J Chem Phys 1943;11(11):521-526.

46. Flory PJ. Principles of Polymer Chemistry. New York: Cornell University Press, Ithaca; 1953. 
47. Nicolson PC, Vogt J. Soft Contact Lens Polymers: an Evolution. Biomaterials 2001;22(24):3273-3283.

48. Hoch G. Modeling of Soft Contact Lens Dehydration [M.S. Thesis]. Berkeley: University of California; 2004.

49. Cheng L, Muller SJ, Radke CJ. Wettability of Silicone-Hydrogel Contact Lenses in the Presence of Tear-Film Components. Curr Eye Res 2004;28(2):93-108.

50. Kohler JE, Flanagan GW. Clinical Dehydration of Extended Wear Lenses. Int Contact Lens Clinic 1985;12(3):152-157.

51. Pritchard N, Fonn D. Dehydration, Lens Movement and Dryness Ratings of Hydrogel Contact-Lenses. Ophthal Physl Opt 1995;15(4):281-286.

52. Rodriguez O, Fornasiero F, Arce A, Radke CJ, Prausnitz JM. Solubilities and Diffusivities of Water Vapor in Poly(Methylmethacrylate), Poly(2Hydroxyethylmethacrylate), Poly(N-vinyl-2-Pyrrolidone) and Poly(acrylonitrile). Polymer 2003;44(20):6323-6333.

53. Malak M, Hill DJT, Whittaker AK. Water Sorption into Poly[(2-Hydroxyethyl Methacrylate)-co-(1-Vinyl-2-Pyrrolidone)] at $310 \mathrm{~K}$. Polymer Int 2003;52(11):17401748.

54. Malak M, Hill DJT, Whittaker AK. Water Sorption into Poly[(2-Hydroxyethyl Methacrylate)-co-(1-Vinyl-2-Pyrrolidone)] at $310 \mathrm{~K}$ (vol 52, pg 1740, 2003). Polymer Int 2004;53(2):235-235 .

55. Kaplan H, Guner A. Characterization and Determination of Swelling and Diffusion Characteristics of poly(N-vinyl-2-pyrrolidone) Hydrogels in Water. J Appl Polym Sci 2000;78(5):994-1000. 
56. Kunzler JF. Silicone-Based Hydrogels for Contact Lens Application. Contact Lens Spectrum 1999;14(8):9s-11s supplement.

57. Favre E, Schaetzel P, Nguygen QT, Clement R, Neel J. Sorption, Diffusion and Vapor Permeation of Various Penetrants through Dense Poly(Dimethylsiloxane) Membranes - A Transport Analysis. J Membrane Sci 1994;92(2):169-184.

58. Watson JM, Baron MG. The Behaviour of Water in Poly(dimethylsiloxane). J Membrane Sci 1996;110(1):47-57.

59. Crank J. The Influence of Concentration-Dependent Diffusion on Rate of Evaporation. P Phys Soc Lond B 1950;63(367):484-491.

60. Crank J. The Mathematics of Diffusion. Oxford: Clarendon Press; 1975.

61. Cairncross RA, Durning CJ. A Model for Drying of Viscoelastic Polymer Coatings. AIChE J 1996;42(9):2415-2425.

62. Nooney GC. Anomalous Diffusion-Controlled Evaporation. J Chem Soc-Faraday Trans 1973(3):330-331.

63. Vinjamur M, Cairncross RA. Non-Fickian Nonisothermal Model for Drying of Polymer Coatings. AIChE J 2002;48(11):2444-2458.

64. Edwards DA. An Asymptotic Analysis of Polymer Desorption and Skinning. Macromol Theor Simul 1999;8(1):10-14.

65. Romdhane IH, Price PE, Miller CA, Benson PT, Wang S. Drying of Glassy Polymer Films. Ind Eng Chem Res 2001;40(14):3065-3075.

66. Opdahl A, Kim SH, Koffas TS, Marmo C, Somorjai GA. Surface Mechanical Properties of pHEMA Contact Lenses: Viscoelastic and Adhesive Property Changes on Exposure to Controlled Humidity. J Biomed Mater Res - A 2003;67A(1):350-356. 
67. Koffas TS, Opdahl A, Marmo C, Somorjai GA. Effect of Equilibrium Bulk Water Content on the Humidity-Dependent Surface Mechanical Properties of Hydrophilic Contact Lenses Studied by Atomic Force Microscopy. Langmuir 2003;19(8):3453-3460.

68. Langel C. personal communication, to be published.

69. Young G, Efron N. Characteristics of the Pre-Lens Tear Film during Hydrogel Contact Lens Wear. Ophthalmic Physiol Opt 1991;11(1):53-8.

70. Guillon JP, Guillon M. Tear Film Examination of the Contact Lens Patient. Optician 1993;206(October 1):21-29.

71. Hamano H. The Change of Precorneal Tear Film by the Application of Contact Lenses. Contact Intraocul Lens Med J 1981; 7( 3): 205-9.

72. Miller KL, Lin M, Radke CJ, Polse KA. Tear Mixing Under Soft Contact Lenses. In: Sweeney DF, editor. Silicone Hydrogels. 2nd ed. Oxford: Butterworth- Heinemann; 2004. p. 57-89.

73. Korb DR, Greiner JV, Herman JP, Hebert E, Finnemore VM, Exford JM, et al. Lid-Wiper Epitheliopathy and Dry-Eye Symptoms in Contact Lens Wearer. CLAO J 2002;24(4):211-216.

74. Meyers JP, Newman J. Simulation of the Direct Methanol Fuel Cell - II. Modeling and Data Analysis of Transport and Kinetic Phenomena. J Electrochem Soc 2002;149(6):A718-A728.

75. Newman J. Electrochemical Systems. 2nd ed. Englewood Cliffs, New Jersey: Prentice Hall, Inc.; 1991. 
76. Press WH, Flannery BP, Teukolsky SA, Vetterling WT. Numerical Recipes in FORTRAN 77. The Art of Scientific Computing. 2nd ed: Cambridge University Press; 1993.

77. Bird RB, Stewart WE, Lightfoot EN. Transport Phenomena. New York: John Wiley and Sons, Inc.; 2002.

78. Ellis B. CRC Polymers: A Properties Database. Sheffield University, UK: Chapman \& Hall / CRC press; 2000. 


\section{Figure Captions}

Fig. 1 Schematic of evaporation-cell apparatus (right) along the humidified air supply (left). The drawing is not to scale.

Fig. 2 Measured steady-state water fluxes as a function of air-flow Reynolds number for HEMA at three relative humidities. Open circles correspond to experimental data at $R H=$ $80 \%$, filled squares at $R H=50 \%$, and open triangles at $R H=20 \%$. Fitted straight lines are also shown. The dotted line is for $R H=80 \%$, the continuous line for $R H=50 \%$, and the dashed line for $R H=20 \%$.

Fig. 3 Steady-state water fluxes as a function of air-flow Reynolds number for SofLens ${ }^{\mathrm{TM}}$ One Day at three relative humidities. Symbols and lines have the same meaning as in Fig. 2.

Fig. 4 Steady-state water-fluxes as a function of air-flow Reynolds number for NTPureVision $^{\mathrm{TM}}$ at three relative humidities. Symbols and lines have the same meaning as in Fig. 2.

Fig. 5 Steady-state water fluxes as a function of air-flow Reynolds number for STPureVision $^{\mathrm{TM}}$ at three relative humidities. Symbols and lines have the same meaning as in Fig. 2. 
Table 1 Soft-contact-lens (SCL) properties

\begin{tabular}{ccccccc}
\hline SCL & Material & $\rho_{p}\left(\mathrm{~g} \mathrm{~cm}^{-3}\right)$ & $L_{0}(\mu \mathrm{m})$ & $w_{10}(\%)$ & $\chi$ & $T_{\mathrm{g}}\left({ }^{\circ} \mathrm{C}\right)$ \\
\hline HEMA & HEMA & $1.274^{\mathrm{b}}$ & $195( \pm 3)$ & 38 & 0.820 & 116 \\
SofLens $^{\mathrm{TM}}$ One Day & Hilafilcon A & 1.28 & $189( \pm 9)$ & 70 & 0.544 & 143 \\
& & & $181( \pm 3)^{\mathrm{c}}$ & 36 & 0.886 & $124^{\mathrm{e}}$ \\
PureVision $^{\text {TM a }}$ & Balafilcon A & 1.11 & $183( \pm 2)^{\mathrm{d}}$ & & & \\
\hline
\end{tabular}

${ }^{\text {a }}$ Both surface plasma treated (ST) and not treated (NT)

${ }^{\mathrm{b}}$ From reference [78]

${ }^{\mathrm{c}}$ For NT-PureVision ${ }^{\mathrm{TM}}$

${ }^{\mathrm{d}}$ For ST-PureVision ${ }^{\mathrm{TM}}$

e Estimated by extrapolating $T_{g}$ vs. $w_{1}$ data to $w_{1}=0$. Direct measurement was not possible because PureVision ${ }^{\mathrm{TM}}$ lens material tends to decompose above $100{ }^{\circ} \mathrm{C}$ [39]. 
Table 2 Steady-state water-fluxes and diffusivities at $24.0{ }^{\circ} \mathrm{C}$ in soft-contact-lens materials in the absence of any air-side resistance. $\left\langle w_{1}\right\rangle$ is the average water content in the SCL membrane at steady state; $L_{0}$ and $L$ are initial and steady state membrane thicknesses; $\bigoplus_{l p}$ and $D_{l p}$ are EMS and Fickian diffusivities, respectively. Fluxes are in $\left(10^{-6} \mathrm{~g} \mathrm{~cm}^{-2} \mathrm{~s}^{-1}\right)$ and diffusivities in $\left(10^{-7} \mathrm{~cm}^{2} \mathrm{~s}^{-1}\right)$ units.

\begin{tabular}{|c|c|c|c|c|c|c|c|c|}
\hline \multirow{2}{*}{$\begin{array}{c}\text { Soft } \\
\text { contact lens }\end{array}$} & \multirow{2}{*}{$\begin{array}{l}\text { RH } \\
(\%)\end{array}$} & \multirow{2}{*}{$n_{1}^{\infty}$} & \multicolumn{3}{|c|}{ EMS } & \multicolumn{3}{|c|}{ FICK } \\
\hline & & & $<w_{1}>$ & $\bigoplus_{l p}$ & $\frac{L}{L_{0}}$ & $<w_{1}>$ & $D_{1 p}$ & $\frac{L}{L_{0}}$ \\
\hline \multirow{3}{*}{ HEMA } & 20 & $4.66( \pm 0.06)$ & 0.16 & 2.59 & 0.702 & 0.20 & 1.66 & 0.733 \\
\hline & 50 & $4.70( \pm 0.06)$ & 0.20 & 3.70 & 0.743 & 0.23 & 2.12 & 0.769 \\
\hline & 80 & $4.45( \pm 0.04)$ & 0.27 & 7.25 & 0.822 & 0.28 & 3.38 & 0.841 \\
\hline \multirow{3}{*}{$\begin{array}{l}\text { SofLens }^{\mathrm{TM}} \\
\text { One Day }\end{array}$} & 20 & $47.1( \pm 1.0)$ & 0.27 & 8.24 & 0.367 & 0.34 & 5.28 & 0.418 \\
\hline & 50 & $65.7( \pm 4.3)$ & 0.32 & 16.0 & 0.404 & 0.38 & 9.23 & 0.450 \\
\hline & 80 & $70.4( \pm 17.2)$ & 0.42 & 34.5 & 0.486 & 0.47 & 15.9 & 0.529 \\
\hline \multirow{3}{*}{$\begin{array}{c}\text { NT- } \\
\text { PureVision }^{\mathrm{TM}}\end{array}$} & 20 & $8.89( \pm 0.14)$ & 0.16 & 5.46 & 0.750 & 0.19 & 3.56 & 0.779 \\
\hline & 50 & $7.95( \pm 0.11)$ & 0.20 & 6.94 & 0.789 & 0.22 & 4.08 & 0.812 \\
\hline & 80 & $9.49( \pm 0.21)$ & 0.26 & 17.3 & 0.860 & 0.27 & 8.38 & 0.873 \\
\hline \multirow{3}{*}{$\begin{array}{c}\text { ST- } \\
\text { PureVision }^{\mathrm{TM}}\end{array}$} & 20 & $8.12( \pm 0.22)$ & 0.16 & 5.04 & 0.750 & 0.19 & 3.29 & 0.777 \\
\hline & 50 & $8.05( \pm 0.37)$ & 0.20 & 7.11 & 0.789 & 0.22 & 4.18 & 0.811 \\
\hline & 80 & $8.55( \pm 0.23)$ & 0.26 & 15.8 & 0.861 & 0.27 & 7.63 & 0.873 \\
\hline
\end{tabular}


Table 3 Effect of evaporative cooling at the membrane/air interface on water/membrane diffusion rates. $\Delta T=T_{L}-T_{0}$ is the difference of interfacial $\left(T_{L}\right)$ and bulk temperatures $\left(T_{0}\right)$, and $\Delta n_{1}=n_{1, \text { sso }}-n_{1}$ is the difference of water fluxes for the isothermal $\left(n_{1, \text { iso }}\right)$ and non-isothermal case $\left(n_{1}\right)$.

\begin{tabular}{cccc}
\hline Soft contact lens & $R H(\%)$ & $\Delta T\left({ }^{\circ} \mathrm{C}\right)$ & $\Delta n_{1} / n_{1, \text { sso }}(\%)$ \\
\hline \multirow{2}{*}{ HEMA } & 20 & -0.81 & 2.67 \\
& 50 & -0.32 & 1.06 \\
\hline & 80 & -0.16 & 0.52 \\
\hline \multirow{2}{*}{$\begin{array}{c}\text { SofLens } \\
\text { one-day }\end{array}$} & 20 & -3.69 & 9.64 \\
\hline & 50 & -1.62 & 3.26 \\
\hline & 80 & -0.58 & 0.77 \\
\hline \multirow{2}{*}{ NT-PureVision } & & & 4.70 \\
& 20 & -1.48 & 1.67 \\
\hline
\end{tabular}


Fig. 1

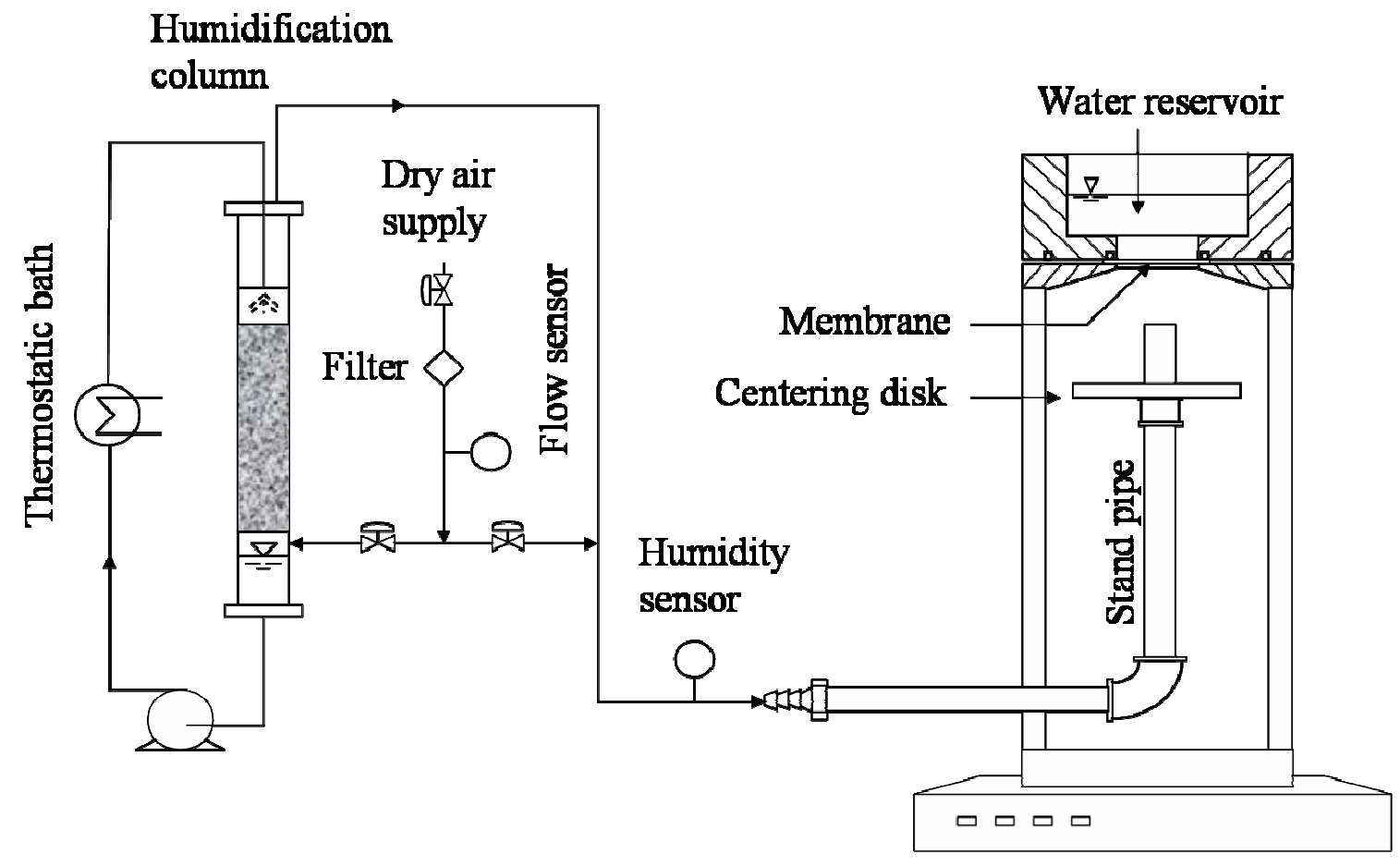

Electronic balance 
Fig. 2

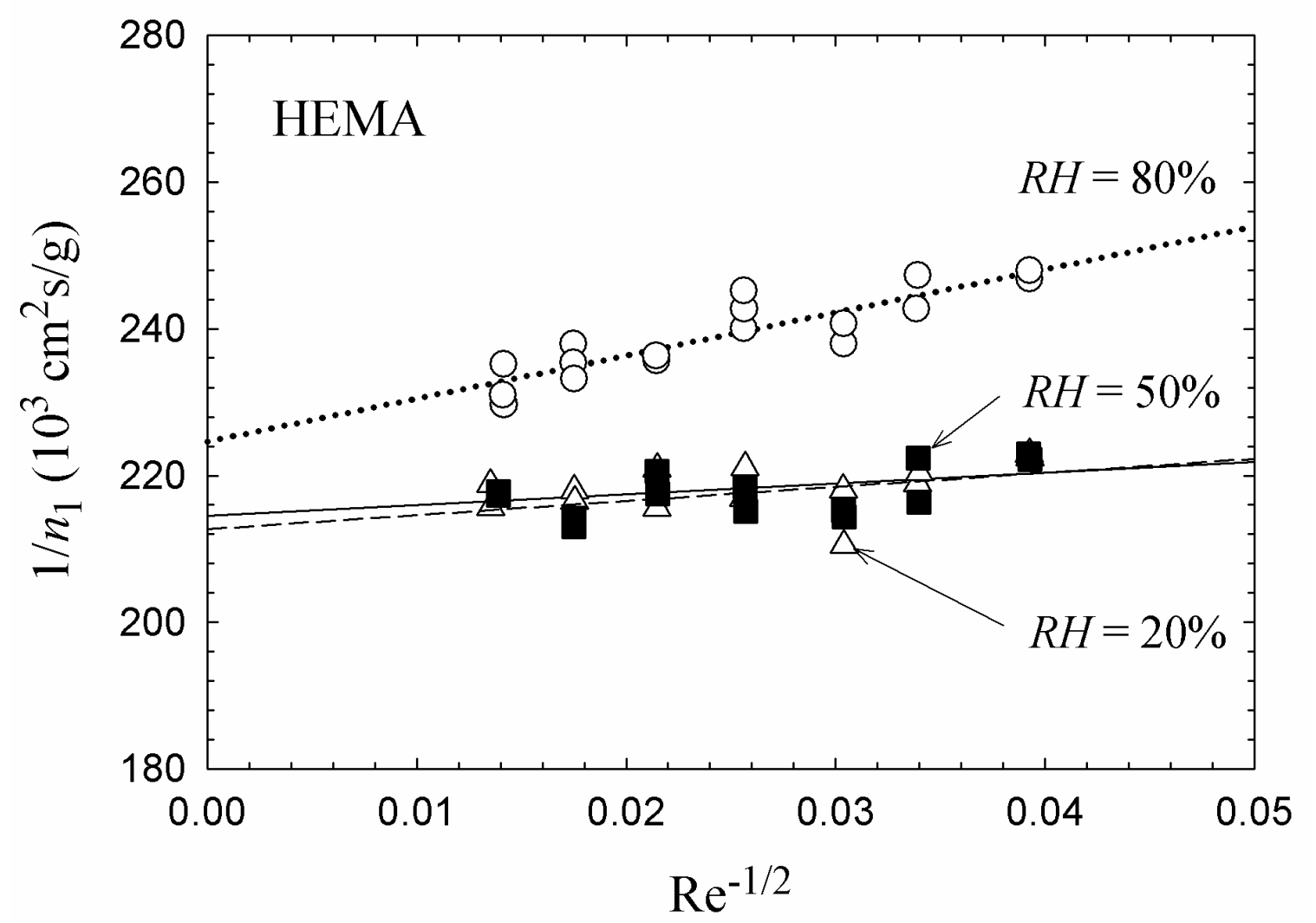


Fig. 3

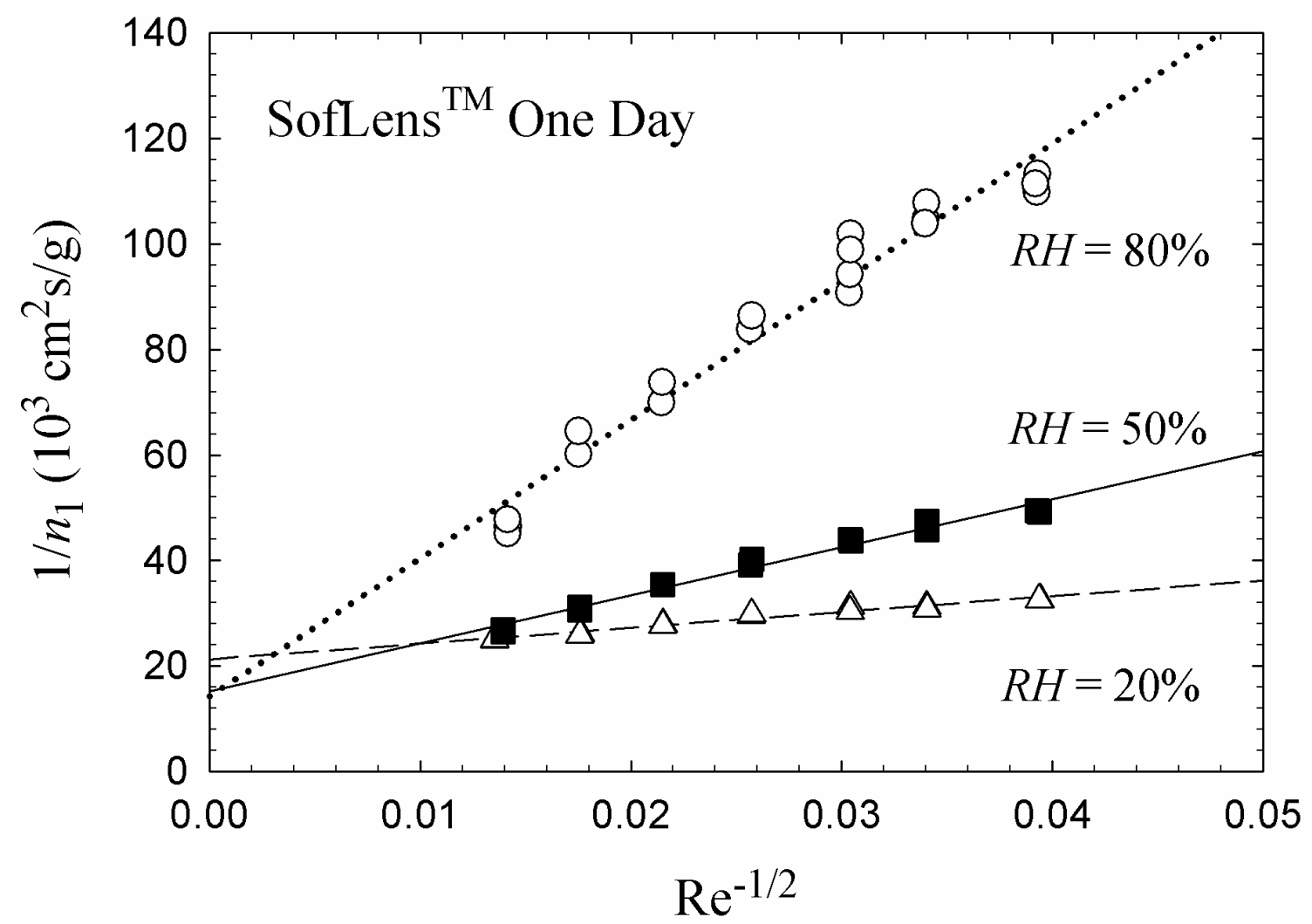


Fig. 4

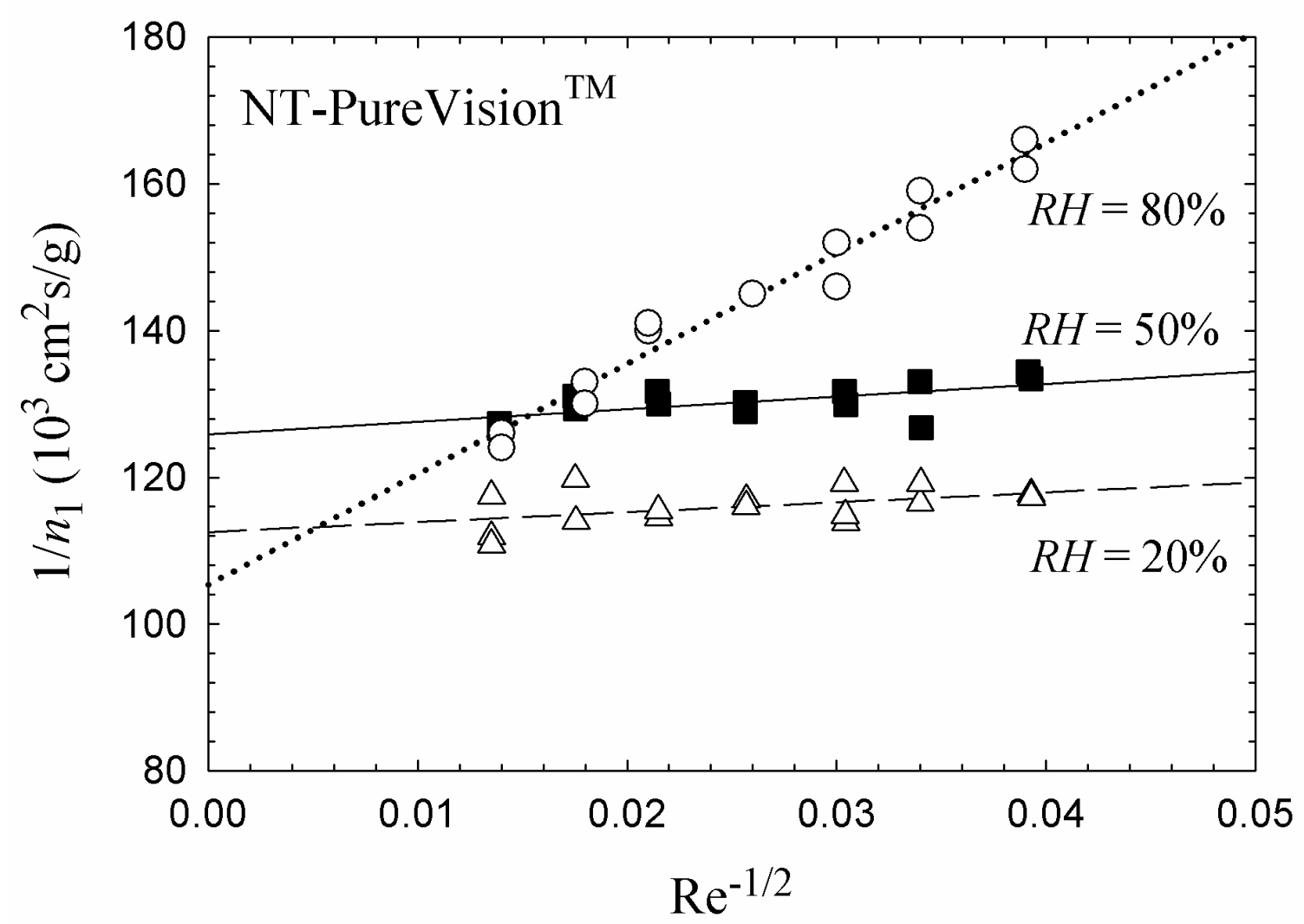


Fig. 5

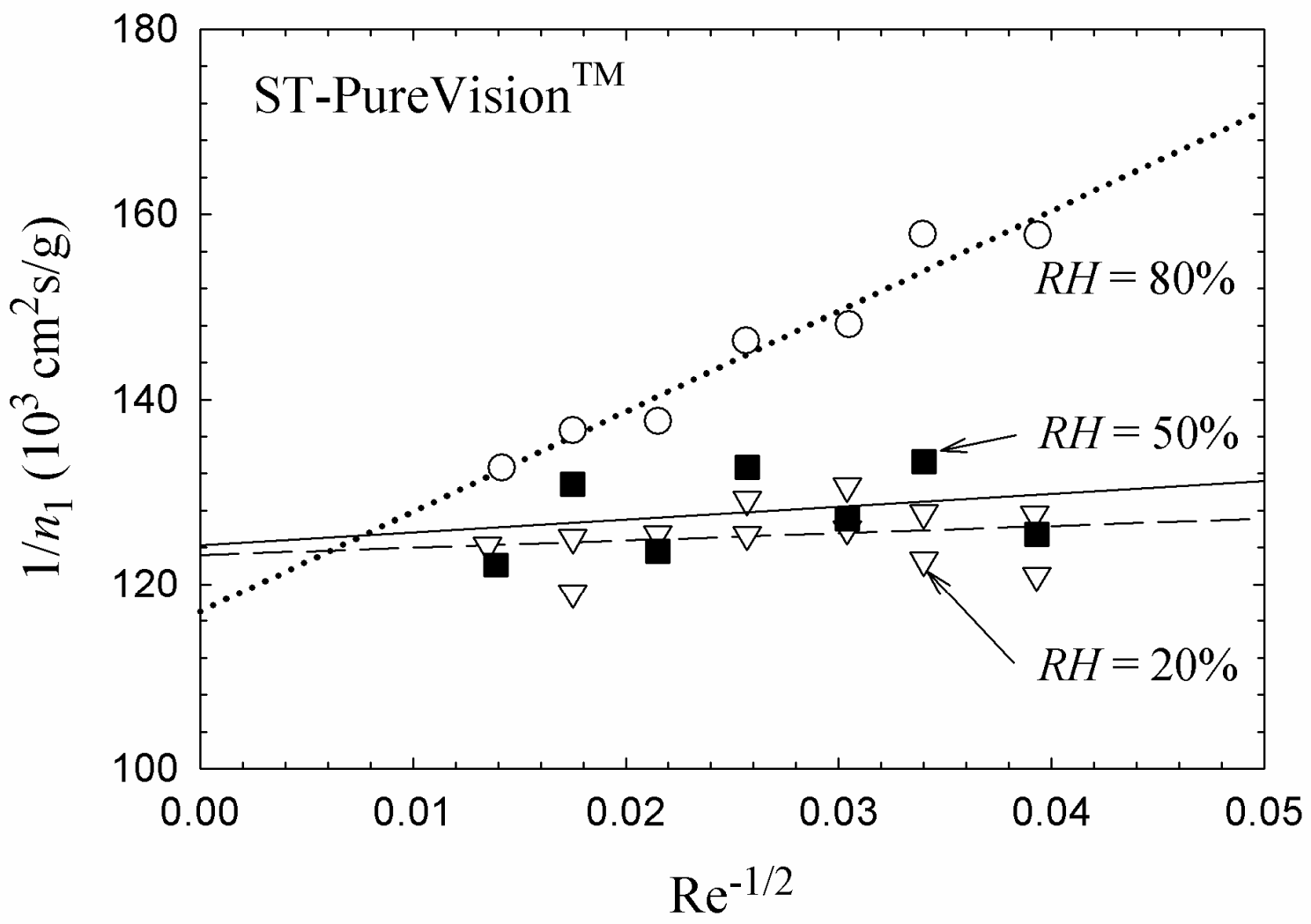

